\title{
Selective relay of afferent sensory-induced action potentials from peripheral nerve to brain and the effects of electrical stimulation
}

\author{
Vijay Sadashivaiah, Pierre Sacré, Yun Guan, William S. Anderson, and Sridevi V. Sarma, IEEE Member
}

\begin{abstract}
Electrical stimulation of peripheral nerve fibers and dorsal column fibers is used to treat acute and chronic pain. Recent studies have shown that sensitized A-fibers maybe involved in the relay of pain information. These nerve fibers also carry sensory-induced action potentials (APs), such as proprioception, mechanoreception, etc. Electrical stimulation of these nerve fibers can result in interactions between sensoryinduced APs and stimulation-induced APs. For example, the sensory-induced APs can collide with stimulus APs, and thus may never be relayed to the brain. In this study, we aimed to quantify the effects of stimulation frequency on these interactions. Specifically, we focused on the goal of stimulation to simultaneously (i) block noxious sensory signals while (ii) relaying innocuous sensory signals from the periphery to the brain via a myelinated nerve fiber. We defined a performance metric called the "selective relay $(S R)$ " measure. Specifically, we constructed a tractable model of a nerve fiber that receives two inputs: the underlying sensory activity at the bottom of the fiber (noxious or innocuous), and the external stimulus applied to the middle of the fiber. We then defined relay reliability, $R$, as the percentage of sensory APs that make it to the top of the fiber. $S R$ is then a product of relaying innocuous sensory information while blocking noxious pain stimuli, i.e., $S R=R_{\text {sen }}\left(1-R_{\text {pain }}\right)$. We applied the two inputs to the fiber at various frequencies and analyzed relay reliability and then we studied selective relay assuming noxious and innocuous stimuli produce APs with distinct frequencies. We found that frequency stimulation between $50-100 \mathrm{~Hz}$ effectively blocks relay of low-frequency pain signals, allowing mid-to-high frequency sensory signals to transmit to the brain.
\end{abstract}

\section{INTRODUCTION}

Acute pain is an early-warning sensory signal triggered in the nervous system, essential to detect and minimize contact with damaging or noxious stimuli. However, the nervous system responsible for acute nociceptive pain processing is fragile as inflammation, nerve injury, and malfunction of the nervous system may divert its function, creating a debilitating disease known as chronic pain. Chronic pain is defined as pain that lasts beyond the time it takes to heal a wound or longer than 12 weeks, and affects about 100 million American adults, more than the total affected by heart disease, cancer, and diabetes combined [1].

Perceived pain intensity is subjective, and under normal conditions, it varies in a sigmoidal fashion in response to

V. Sadashivaiah, P. Sacré and S. V. Sarma are with the Institute for Computational Medicine and the Department of Biomedical Engineering, The Johns Hopkins University, Baltimore, MD (vjs@jhu.edu, p.sacre@jhu.edu, sree@jhu.edu).

Y. Guan is with the Department of Anesthesiology/Critical Care Medicine, The Johns Hopkins University School of Medicine, Baltimore, MD (yguan1@jhmi.edu).

W. S. Anderson is with the Institute for Computational Medicine and the Department of Neurosurgery, The Johns Hopkins University School of Medicine, Baltimore, MD (wanders5@jhmi.edu). stimulus intensity [2]. However, in chronic pain conditions, the system is more sensitive to pain (hyperalgesia) for less provocative or innocuous stimulus (allodynia) [3] (see Fig. 1A). This is known as a process that involves essential central sensitization. Chronic pain is primarily treated with drugs, which have negative side effects and lose efficacy over long-term use [4], [5]. Apart from addiction, studies have shown that chronic and acute exposure to opioids can lead to sensitization of the pain system [6]. Alternatively, chronic pain is also treated with electrical neurostimulation, which has fewer side effects. Although effective, electrical neurostimulation achieves suboptimal efficacy over longterm use as the underlying mechanisms remain unclear.

Critical to advancing chronic pain treatment is a deeper mechanistic understanding of pain transmission and modulation under both normal and pathological conditions, which remain largely elusive because the pain system is complex and builds on a tightly regulated dynamical crosstalk between the peripheral nervous system and the brain via the spinal cord. Under trauma or injury, painful stimuli picked up by peripheral nociceptors and generate action potentials (APs) that travel through the peripheral $\mathrm{A} \delta$ (fast) and $\mathrm{C}$ (slow) fibers into the dorsal root ganglion. These pain signals then travel up the spinothalamic tract via projection neurons to reach the supraspinal centers, where the pain is ultimately perceived. On the other hand, sensory stimuli such as touch and proprioception are picked up by peripheral innocuous receptors which generate APs that travel through the peripheral $\mathrm{A} \alpha$ and $\mathrm{A} \beta$ fibers into the dorsal horn. These sensory signals then travel up the dorsal column in medial lemniscus pathway to reach the thalamus and sensory cortex.

Under normal conditions, both pain and sensory pathways mediate information in a relatively independent fashion. However, under pathological conditions, sensory pathways may pick up pain signals. Recent studies suggest that, under a sensitized condition (see Fig. 1B), A-fiber mechanoreceptors gain abnormal access to the nociceptive pathway and can induce pain [8], [9]. This suggests that both innocuous and noxious stimuli may travel along the same fibers up to the brain.

Over the past decade, computational efforts have moved towards modeling the effects of electrical neurostimulation in nerve fibers [10]. Detailed mechanistic models have been constructed and compared to experimental data and are reviewed in [11]. Although it is important to understand how different electrode configurations and stimulation parameters alter the electric field produced near the fibers, current models do not consider sensory signals traveling along the 


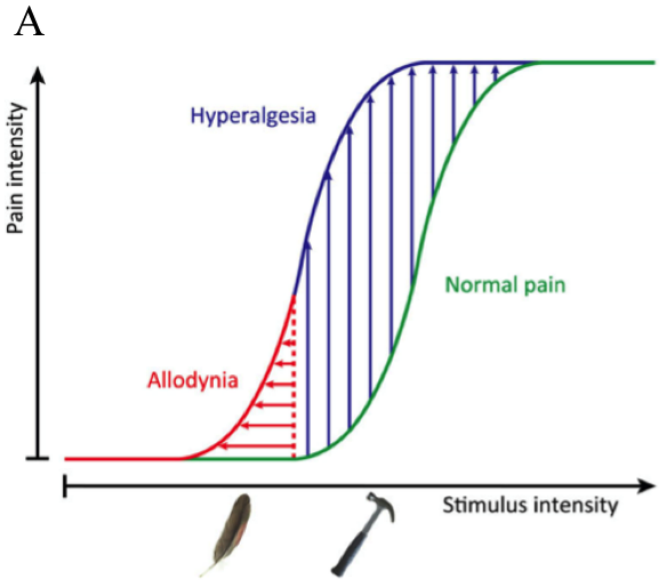

B

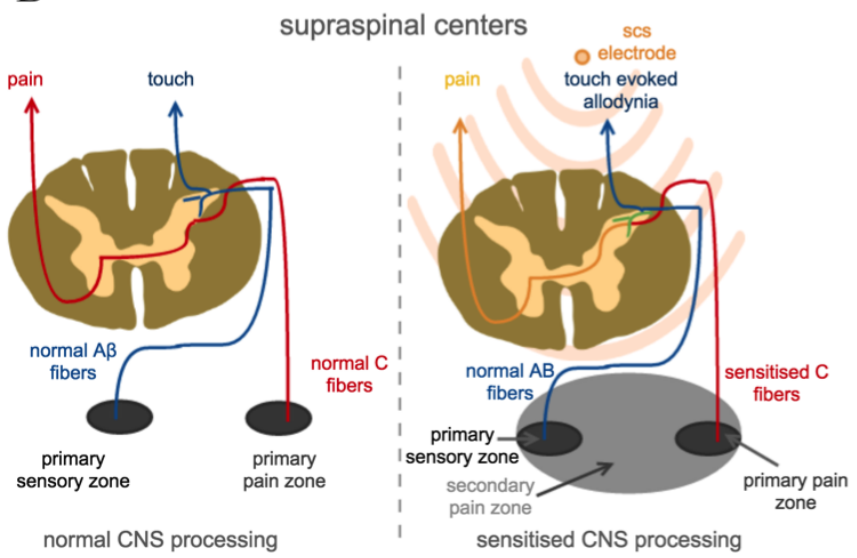

Fig. 1. Pain sensitization and mechanical hypersensitivity. A. Illustration of pain sensitization under chronic pain conditions. Under normal condition, perceived pain would follow the green curve. However, under sensitized conditions we perceive more pain than normal (hyperalgesia) for a less provocative stimulus (allodynia). These are pictured in blue and red curve respectively. Extracted and re-drawn from [7] B. Pain and sensory signal processing in central nervous system. Under normal conditions, painful stimuli are picked up by peripheral nociceptors which travel up through slow $\mathrm{C}$ fibers to reach the supraspinal centers via spino-thalamic tract. On the other hand, sensory signals travel up the fast A-delta and A-beta fibers via medial lemniscus pathway to reach supraspinal centers. Recent studies suggest that, under a sensitized condition, A-fiber mechanoreceptors gain abnormal access to the nociceptive pathway and induce pain. When spinal cord stimulation is applied we end up affecting both pain and sensory pathways. Ideally we want to selectively block pain but relay sensory information.

same fibers, which is relevant to chronic pain subjects with central sensitization. Specifically, none of the existing computational models incorporate underlying sensory activity traveling along the fibers when studying responses to electrical neurostimulation. Therefore, these models do not capture the interactions (e.g. collisions of stimulationinduced APs) between the stimulus-induced APs and sensory initiated APs along the fibers.

In this study, we construct a tractable model of a single nerve fiber that incorporates both sensory and stimulation inputs. The reduced model is a simplified model of a myelinated nerve fiber that captures the interactions of sensory and stimulus inputs based on the speed of conduction, refractory periods and activation thresholds. The model receives two inputs: the underlying sensory activity at the bottom end of the fiber and the external stimulus applied to the middle of the fiber. In particular, we model the sensory input as a Poisson distribution and the stimulus as a deterministic periodic input. We then compute relay reliability, $R$, defined as the ratio of the number of sensory APs that make it to the top of the fiber over the total number of sensory APs entering the bottom of the fiber. In this study, we vary the frequency of the input signals and analyze relay reliability and then selective relay of innocuous signals (while blocking noxious signals) under different settings.

\section{Methods}

\section{A. Myelinated nerve fiber}

A myelinated nerve fiber is a cylindrical active membrane (axon), tightly wrapped in an insulating myelin sheath. This myelin sheath is interrupted periodically, leaving short gaps where the axonal membrane is exposed. In our reduced model, the nerve fiber is characterized by its geometry (length and diameter) and three macroscopic properties: conduction velocity, refractory period, and the activation threshold of the fiber. These macroscopic properties can be estimated either from biological experiments or from our high-dimensional model [12]. Specifically, we assume the following.

- A stimulation or sensory input creates an action potential in the fibers if and only if its amplitude is larger than the associated threshold and its timing is not within the refractory of a preceding action potential.

- An action potential propagates in the fiber in both directions at a constant velocity (the conduction velocity).

- The interaction of an orthodromic and antidromic action potentials results in a collision, i.e., the annihilation of both action potentials. Based on these characteristics, we can identify the interactions occurring in the fiber based only on the timing of sensory and stimulation pulses [13].

\section{B. Electrical potential field generated by the stimulation}

The extracellular medium surrounding a nerve fiber is composed of different regions of the spinal cord (epidural fat, cerebrospinal fluid, white matter, grey matter, etc.), which have different conduction properties [14]. In addition, the electrode can also take various shapes (single contact, array of contact, etc.) and various configurations (monopolar, bipolar, etc.) [15]. But for the sake of reduced model, we assume that the extracellular stimulus produces periodic spike train at the middle of the myelinated nerve fiber. We consider stimulation frequencies ranging from 0 to $200 \mathrm{~Hz}$.

\section{Underlying sensory activity}

The dorsal column contains nerve fibers that relay peripheral sensory inputs to supraspinal centers. In normal conditions, these myelinated nerve fibers that originate in the low-threshold primary sensory neurons that mostly transmit non-noxious sensory stimuli: proprioception from skeletal muscles and mechanoreception from the skin. 
However, in pathological conditions, mechanical hypersensitivity after injury may also be signaled by abnormal activity in dorsal column fibers [8], [9]. Therefore, the spiking activity in these fibers spans a broad frequency range and exhibits various patterns [16]: regular spike discharge, regular discharge of doublet spikes, bursting patterns, sporadic activity with no regular or predictable firing pattern, etc.

The presence of underlying sensory activity in the nerve fiber is represented by replacing a 'sealed-end' boundary condition by a current source at one end of the nerve fiber. As a first step, the underlying sensory activity is modeled as a Poisson spike train. Therefore, the instantaneous firing rate $\lambda^{\text {sen }}$ is assumed constant, ranging from 0 to $200 \mathrm{~Hz}$.

\section{Selective relay}

We define reliability as,

$$
R\left(\lambda_{\text {stim }}, \lambda_{\text {sen }}\right)=\frac{\text { number of relayed sensory pulses }}{\text { total number of sensory pulses }},
$$

where relayed sensory inputs are sensory inputs that travel from the bottom to the top of the fiber. Depending on the sensory origin of the underlying sensory activity, we may want to modulate its relay to the top of the fiber differently with electrical stimulation.

For example, we want to block pathological sensory activity (as pain), but we also want to keep relaying normal sensory activity (as proprioception or mechanoreception) in neighboring fibers also affected by the electric field. To achieve this, we define the following performance metric:

$$
S R\left(\lambda_{\text {stim }}, \lambda_{\text {sen }}, \lambda_{\text {pain }}\right)=R_{\text {sen }} \times\left[1-R_{\text {pain }}\right] .
$$

$S R$ is the product of reliability of sensory information and the blocking of pain signal. We are interested in maximizing the sensory AP relay while minimizing the pain AP relay. Thus, our goal is to maximize $S R$ with appropriately tuned stimulation. We consider three frequency ranges for the pain signal input: low $(1-50 \mathrm{~Hz})$, medium $(51-151 \mathrm{~Hz})$ and high $(152-200 \mathrm{~Hz})$.

\section{RESULTS}

In this section we describe the results from our simulation testbed. We consider three different diameters of nerve fibers in our simulations $(6 \mu \mathrm{m}, 9 \mu \mathrm{m}$ and $12 \mu \mathrm{m})$. These results are drawn from 50 simulations for each frequency pair (sensory, stimulus) of this model with a stochastic Poisson physiological input. Analysis of data and reduced model simulations were performed on MATLAB, MathWorks.

For each frequency range of the pain signal, the average $S R$ is plotted against the stimulus frequency. This is repeated for all the diameters under consideration (see Fig. 2). We describe results in more details for the $9 \mu \mathrm{m}$-diameter nerve fiber.

- Low pain frequency $(1-50 \mathrm{~Hz}): S R$ increases for all sensory frequency ranges. After reaching a maximum value, there is a localized decrease. We see that the maximum $S R$ occurs at 0.32 for high sensory frequency

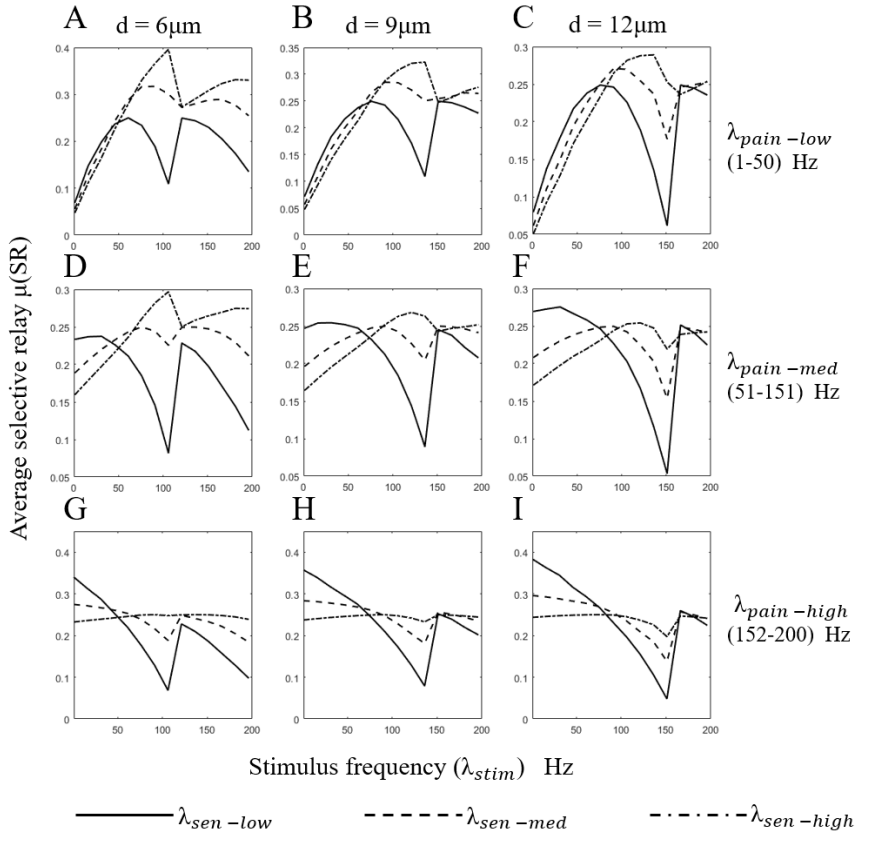

Fig. 2. Optimizing spinal cord stimulation to maximize selective reliability. Here we plot the selective reliability $(S R)$ against stimulus frequency for different diameters. Our goal is to maximize $S R$, i.e. maximize relay of sensory APs while blocking stimulus APs. We divide the frequency into 3 ranges, low $(1-50 \mathrm{~Hz})$, medium $(51-151 \mathrm{~Hz})$ and high $(152-200 \mathrm{~Hz})$. A, D \& G. For each pain frequency range (low, medium \& high), we plot $S R$ for different sensory frequency ranges (low, medium \& high). Here we considered $6 \mu \mathrm{m}$ diameter nerve fiber. B, E, H \& C, F, I. Similar plots for $9 \mu \mathrm{m}$ and $12 \mu \mathrm{m}$ diameter nerve fibers.

at a stimulation frequency of $135 \mathrm{~Hz}$. Fig. 2B also shows that the stimulus frequency corresponding to maximum $S R$ value shifts to the right as the sensory frequency increases. The trend follows,

$S R\left(\lambda_{\text {stim }}, \lambda_{\text {sen }}^{\text {high }}, \lambda_{\text {pain }}^{\text {low }}\right)>S R\left(\lambda_{\text {stim }}, \lambda_{\text {sen }}^{\text {med }}, \lambda_{\text {pain }}^{\text {low }}\right)>$ $S R\left(\lambda_{\text {stim }}, \lambda_{\text {sen }}^{\text {low }}, \lambda_{\text {pain }}^{\text {low }}\right)$.

- Medium pain frequency $(51-151 \mathrm{~Hz})$ : We see a similar trend in $S R$ values as in the previous low pain frequency case. Although the maximum $S R$ value 0.27 achieved for $120 \mathrm{~Hz}$ stimulation is lower, the trend follows, $S R\left(\lambda_{\text {stim }}, \lambda_{\text {sen }}^{\text {high }}, \lambda_{\text {pain }}^{\text {med }}\right)>S R\left(\lambda_{\text {stim }}, \lambda_{\text {sen }}^{\text {low }}, \lambda_{\text {pain }}^{\text {med }}\right)>$ $S R\left(\lambda_{\text {stim }}, \lambda_{\text {sen }}^{\text {med }}, \lambda_{\text {pain }}^{\text {med }}\right)$.

- High pain frequency $(152-200 \mathrm{~Hz}): S R$ starts high for low stimulus frequencies and then decreases to a minimum, but then increases again after the zero band. The maximum $S R$ value 0.36 is achieved at $1 \mathrm{~Hz}$ stimulation for a low sensory frequency band. The trend follows, $S R\left(\lambda_{\text {stim }}, \lambda_{\text {sen }}^{\text {low }}, \lambda_{\text {pain }}^{\text {high }}\right)>\operatorname{SR}\left(\lambda_{\text {stim }}, \lambda_{\text {sen }}^{\text {med }}, \lambda_{\text {pain }}^{\text {high }}\right)>$
$S R\left(\lambda_{\text {stim }}, \lambda_{\text {sen }}^{\text {high }}, \lambda_{\text {pain }}^{\text {high }}\right)$.

From Fig. 2, it is evident that using a low frequency stimulation is effective against a low pain frequency, while medium frequency stimulation is effective for low and medium frequency pain signals. Higher frequency stimulation may effectively block pain signal relay, but also hinders the relay of sensory information. 


\section{DISCUSSION}

Electrical neurostimulation may selectively modulate the relay of action potentials in the nerve fiber. To quantify this, we defined a selective relay metric, $S R$. When the pain signal is low frequency, $S R$ increases for all sensory frequency ranges as a function of stimulus frequency, and after reaching a maximum value, there is a localized decrease of $S R$. The stimulus frequency corresponding to maximum value of $S R$ shifts to the right as the sensory frequency increases and the value of $S R$ itself increases. This is expected, as higher sensory frequencies result in a higher selective relay.

We see a similar trend in the medium pain frequency range, but the maximum $S R$ is lower. Effectively, with a higher pain frequency, it becomes more difficult to block all the noxious APs. When the pain frequency is high, the $S R$ value is highest when the stimulus frequency is very low. This is because, at high pain frequency, most noxious generated APs block themselves resulting in a high $S R$. All images in Fig. 2 show that a maximum selective relay is achieved at a low frequency of $50-100 \mathrm{~Hz}$, which is, interestingly, what is conventionally used in clinical practice treating chronic pain with peripheral nerve or and spinal cord stimulation [17]-[19].

Nerve fibers are the primary target of any electrical neurostimulation treatment. Under pathological conditions, a portion of myelinated $A \beta$ nerve fibers may transmit noxious stimuli alongside useful sensory information. Electrical neurostimulation may end up blocking the useful sensory information while blocking the noxious APs. For example, spinal cord stimulation may reduce activities in dorsal column fibers from reaching second-order neurons in the brainstem, including cells in the gracile nucleus and cuneate nucleus. Thus, spinal cord stimulation may interfere and alter the information coded by innocuous sensory inputs.

Recent studies have shown the use of very high frequency stimulation $(1000-10,000 \mathrm{~Hz})$ to be effective in treating chronic pain [20]. The choice of an optimal stimulation frequency may result from the dual objective to relay a normal sensory input and to block a noxious one. Using high frequency stimulus might saturate the nerve fiber and block all APs from reaching the brain.

Our simulation test bed is the first step toward a better understanding of the effect of neurostimulation in the relay of peripheral afferent inputs in nerve fibers. In the future, we plan (a) to consider more complex sensory inputs than Poisson, such as doublets or bursts, and (b) to augment the dorsal column model to be mechanistic and to include collateral fibers to the dorsal horn and the dorsal horn circuit.

\section{ACKNOWLEDGMENT}

Work supported by NIH R01 AT009401 to S.V.S., Y.G., and W.S.A, and a NPRI Postdoctoral fellowship to P.S. We would like to thank Dr. M. Caterina, Neurosurgery Pain Research Institute, The Johns Hopkins University School of Medicine, for valuable and insightful discussions.

\section{REFERENCES}

[1] L. S. Simon, "Relieving pain in america: A blueprint for transforming prevention, care, education, and research," Journal of Pain \& Palliative Care Pharmacotherapy, vol. 26, no. 2, pp. 197-198, 2012.

[2] Y. Zhang, N. Wang, J.-Y. Wang, J.-Y. Chang, D. J. Woodward, and F. Luo, "Ensemble encoding of nociceptive stimulus intensity in the rat medial and lateral pain systems," Molecular Pain, vol. 7, no. 1, p. 64, Aug 2011.

[3] C. J. Woolf, "Central sensitization: Implications for the diagnosis and treatment of pain," Pain, vol. 152, no. 3S, 2011

[4] H. Breivik, B. Collett, V. Ventafridda, R. Cohen, D. Gallacher, M. B. Löfvander, A. W. Engström, E. Iglesias, L. A. Mitchell, R. A MacDonald et al., "1. survey of chronic pain in europe: Prevalence, impact on daily life, and treatment," European Journal of Pain, vol. 10, no. 4, pp. 287-384, 2006.

[5] R. Chou, "Opioid treatment guide-lines: clinical guidelines for the use of chronic opioid therapy in chronic noncancer pain," J Pain, vol. 10, pp. 113-130, 2009.

[6] F. Marchand, M. Perretti, and S. B. McMahon, "Role of the immune system in chronic pain," Nature Reviews. Neuroscience, vol. 6, no. 7, p. 521, 2005.

[7] S. Lolignier, N. Eijkelkamp, and J. N Wood, "Mechanical allodynia," vol. 467, 052014.

[8] R. Baron, "Neuropathic pain: A clinical perspective," Handbook of experimental pharmacology, vol. 194, pp. 3-30, 2009.

[9] Y. Song, H.-M. Li, R.-G. Xie, Z.-F. Yue, X.-J. Song, S.-J. Hu, and J.L. Xing, "Evoked bursting in injured a $\beta$ dorsal root ganglion neurons: A mechanism underlying tactile allodynia," Pain, vol. 153, no. 3, pp. 657-665, 2012

[10] S. Joucla and B. Yvert, "Modeling extracellular electrical neural stimulation: From basic understanding to mea-based applications," Journal of Physiology-Paris, vol. 106, no. 3, pp. 146-158, 2012.

[11] E. J. Argüello, R. J. Silva, M. K. Huerta, and R. S. Avila, "Computational modeling of peripheral pain: a commentary," Biomedical engineering online, vol. 14, no. 1, p. 56, 2015.

[12] V. Sadashivaiah, P. Sacré, Y. Guan, W. S. Anderson, and S. V. Sarma, "Modeling electrical stimulation of mammalian nerve fibers: A mechanistic versus probabilistic approach," in Engineering in Medicine and Biology Society (EMBC), 2017 39th Annual International Conference of the IEEE. IEEE, 2017, pp. 3868-3871.

[13] A. Gonzalez-Perez, R. Budvytyte, L. D. Mosgaard, S. Nissen, and T. Heimburg, "Penetration of action potentials during collision in the median and lateral giant axons of invertebrates," Physical Review X, vol. 4, no. 3, p. 031047, 2014.

[14] J. J. Struijk, J. Holsheimer, B. Van Veen, and H. B. Boom, "Epidural spinal cord stimulation: calculation of field potentials with special reference to dorsal column nerve fibers," IEEE Transactions on Biomedical Engineering, vol. 38, no. 1, pp. 104-110, 1991.

[15] Medtronic Neuromodulation, Technical design summary: Model 39565 specify 5-6-5 surgical lead, 2007.

[16] K. C. Kajander and G. J. Bennett, "Onset of a painful peripheral neuropathy in rat: A partial and differential deafferentation and spontaneous discharge in $\mathrm{A} \beta$ and $\mathrm{A} \delta$ primary afferent neurons," Journal of Neurophysiology, vol. 68, no. 3, pp. 734-744, 1992.

[17] B. A. Meyerson and B. Linderoth, "Mode of action of spinal cord stimulation in neuropathic pain," Journal of Pain and Symptom Management, vol. 31, no. 4, pp. S6-S12, 2006.

[18] G. Lind, G. Schechtmann, J. Winter, and B. Linderoth, "Drugenhanced spinal stimulation for pain: a new strategy," in Operative Neuromodulation. Springer, 2007, pp. 57-63.

[19] F. Yang, A. F. Carteret, P. W. Wacnik, C.-Y. Chung, L. Xing, X. Dong, R. A. Meyer, S. N. Raja, and Y. Guan, "Bipolar spinal cord stimulation attenuates mechanical hypersensitivity at an intensity that activates a small portion of A-fiber afferents in spinal nerve-injured rats,' Neuroscience, vol. 199, pp. 470-480, 2011.

[20] L. Kapural, C. Yu, M. W. Doust, B. E. Gliner, R. Vallejo, B. T. Sitzman, K. Amirdelfan, D. M. Morgan, T. L. Yearwood, R. Bundschu et al., "Comparison of 10-khz high-frequency and traditional lowfrequency spinal cord stimulation for the treatment of chronic back and leg pain: 24-month results from a multicenter, randomized, controlled pivotal trial," Neurosurgery, vol. 79, no. 5, pp. 667-677, 2016. 